\title{
THE UNIQUENESS OF THE CAUCHY PROBLEM FOR PARTIAL DIFFERENTIAL EQUATIONS WHICH MAY HAVE MULTIPLE CHARACTERISTICS
}

\author{
BY \\ PETER M. GOORJIAN
}

1. Introduction. Nirenberg [1] proved uniqueness of solutions to the Cauchy problem across convex surfaces for equations of order $m$ with constant leading coefficients and with characteristics of multiplicity at most $r, 0<r \leqq m$. Only lower order derivatives of order at most $m-r$ were allowed in the equations. In $\$ 2$ we shall prove uniqueness of the Cauchy problem across certain convex surfaces for equations of order $m$ with constant leading coefficients, with complex characteristics of multiplicity at most $r$ and with real characteristics of multiplicity at most $s$. Only lower order derivatives of order at most $m-q$ are allowed in the equations, where $q$ is the maximum of $s$ and the largest integer no greater than $(r+1) / 2$.

Protter [7] proved uniqueness across arbitrary surfaces for certain elliptic equations of order $m$ with variable leading coefficients, with characteristics of multiplicity $r$ and with lower order derivatives of order no greater than $m-r / 2$. The principal part was derived from the product of $r$ second order elliptic operators. In $\S 4$ we shall generalize these results to allow factors of arbitrary order with simple characteristics, and the equations will have Lipschitz continuous leading coefficients.

In $\S 5$ we shall compare the present results with Cohen's [1] counterexamples. We shall see that if a lower order derivative of order higher than we allow should occur in the equation then uniqueness fails. In $\$ 3$ we shall prove uniqueness across certain convex surfaces for parabolic equations in which the elliptic operator has constant coefficients. Finally, in the above equations for which uniqueness holds, the lower order terms are merely required to have locally bounded, measurable coefficients, whereas Cohen's counterexamples have continuous coefficients. In $\S 6$ we shall prove some special uniqueness results for equations with lower order terms of order greater than previously allowed, provided all coefficients of terms above a certain order are constant, the remaining ones being measurable and bounded.

NotATIONS AND DEFINITIONS. Let $\Omega$ be an open set in real $n$-dimensional Euclidean space $R^{n}, n \geqq 2 ; x=\left(x_{1}, \ldots, x_{n}\right) \in \Omega ; \alpha=\left(\alpha_{1}, \ldots, \alpha_{n}\right)$ is a multi-index where the $\alpha_{k}$ are nonnegative integers; $|\alpha|=\sum_{k=1}^{n} \alpha_{k} . \xi=\left(\xi_{1}, \ldots, \xi_{n}\right) \in R^{n} . \xi^{\alpha}=$ $\xi_{1}^{\alpha_{1}} \ldots \xi_{n}^{\alpha_{n}} . \zeta=\left(\zeta_{1}, \ldots, \zeta_{n}\right) \in C^{n}$, where $\zeta_{k}, k=1, \ldots, n$ are complex numbers and $C^{n}$

Received by the editors May 1, 1969. 
is complex $n$-dimensional Euclidean space. $\zeta^{\alpha}=\zeta_{1}^{\alpha}{ }_{1} \ldots \zeta_{n}^{\alpha_{n}}$. Let $P(x, \zeta)$ be a polynomial of order $m$ in $n$ variables $\zeta_{1}, \ldots, \zeta_{n}$ with complex variable coefficients defined for $x \in \Omega$. $[a]$ is the largest integer less than or equal to $a . k$ is always a single index, $k=1, \ldots, n$.

$D_{k}=-i \partial / \partial x_{k} . D=\left(D_{1}, \ldots, D_{n}\right) . D_{\alpha}=D_{1}^{\alpha_{1}} \ldots D_{n}^{\alpha_{n}}$. Let $P(x, D)$ denote a linear partial differential operator of order $m$ with complex variable coefficients defined for $x \in \Omega$. $P(x, D)$ is obtained by replacing $\zeta_{k}$ with $D_{k}$ in $P(x, \zeta)$; thus $P(x, D)$ $=\sum_{|\alpha| \leqslant m} a_{\alpha}(x) D_{\alpha} \cdot P(x, \zeta)$ is called the symbol associated with $P(x, D)$. The principal part $P_{m}(x, D)$ of $P(x, D)$ is $\sum_{|\alpha|=m} a_{\alpha}(x) D_{\alpha} . P^{(\alpha)}(x, \zeta)=\partial^{|\alpha|} P(x, \zeta) / \partial \zeta_{1}^{\alpha_{1}} \ldots \partial \zeta_{n^{n}}^{\alpha_{n}}$ and the special case when the latin letter $k$ is used $P^{(k)}(x, \zeta)=\partial^{k} P(x, \zeta) / \partial \zeta_{1}^{k}$. When $P(x, D)$ has constant coefficients, then $P(x, D) \equiv P(D)$.

The equation $P_{m}\left(x, \zeta_{1}, \xi_{2}, \ldots, \xi_{n}\right)=0$ is called the characteristic equation for $P(x, D)$, where $\xi_{2}, \ldots, \xi_{n}$ are real and not all zero and $\zeta_{1}$ is complex. Assume the coefficient of $\zeta_{1}^{m}$ is not zero. If the $m$ roots $R_{1}, \ldots, R_{m}$ of the characteristic equation at $x_{0} \in \Omega$ are always distinct for all real choices of $\left(\xi_{2}, \ldots, \xi_{n}\right) \neq 0$, we say that $P(x, D)$ has simple characteristics at $x_{0}$. If for some choice of $\left(\xi_{2}, \ldots, \xi_{n}\right) \neq 0$ we have a (real) root of multiplicity $r$ we say we have a (real) characteristic of multiplicity $r$ at $x_{0}$. If real characteristics do not occur we say $P(x, D)$ is elliptic at $x_{0}$.

\section{Equations with constant leading coefficients and with complex or real character-} istics. We shall study solutions of the differential inequality

$$
|P(D) u| \leqq K \sum_{|\alpha| \leqq m-q}\left|D_{\alpha} u\right|
$$

where $P(D)$ is a homogeneous operator of order $m$ with constant coefficients, with complex characteristics of multiplicity at most $r$ and with real characteristics of multiplicity at most $s, q=\max (s,[(r+1) / 2]), u \in C^{m}$ is a solution of the differential inequality which vanishes for $x_{1} \leqq \varepsilon\left(x_{2}^{2}+\cdots+x_{n}^{2}\right), \varepsilon>0$, when $x$ is in a neighborhood of the origin. We shall prove that $u$ vanishes in a neighborhood of the origin.

The symbol $P(\zeta)$ has the following two properties: the set of polynomials $P^{(k)}(\xi), 0 \leqq k \leqq s, 0 \neq \xi \in R^{n}$ have no common zero, and the set of polynomials $P^{(k)}\left(\zeta_{1}, \xi_{2}, \ldots, \xi_{n}\right), 0 \leqq k \leqq r, 0 \neq\left(\xi_{2}, \ldots, \xi_{n}\right) \in R^{n-1}, \zeta_{1} \in C$ have no common zero.

We drop the restriction on the symbol $P(\zeta)$ that the coefficient of $\zeta_{1}^{m}$ is not zero. Rather, we require the property of $P(\zeta)$ concerning the complex roots to still be valid, and that the multiplicity of the real characteristics of $P(D)$ is at most $s$ in the sense that the set of polynomials $P^{(\alpha)}(\xi)$ with $|\alpha|=s$ have no common zero for $0 \neq \xi \in R^{n}$. By Euler's theorem on homogeneous polynomials, this class of operators will include those previously defined. Also included in this class are operators such as the wave operator in two dimensions $P(D)=-D_{1} D_{2}$, for which the $x_{1}$ axis is a characteristic direction.

We use these two properties of $P$ to prove a key inequality. 
Proposition 1. Let $N_{0}=(-1,0, \ldots, 0), \xi=\left(\xi_{1}, \ldots, \xi_{n}\right) \in R^{n}$ and $\tau \in R$. Then there is an open cone $V \subset R^{n}$ with $N_{0} \in V$ such that for $N \in V$

$$
\begin{aligned}
|\xi+i \tau N|^{2(m-s)} \leqq C\left(\sum_{|\alpha|=s}\left|P^{(\alpha)}(\xi+i \tau N)\right|^{2}\right. & \\
& \left.+\sum_{k=s+1}^{r}(\tau|N|)^{2(k-s)}\left|P^{(k)}(\xi+i \tau N)\right|^{2}\right) .
\end{aligned}
$$

If $r \leqq s$, then there is no second sum in (2.2).

Proof. Because of the continuity and homogeneity properties of $P$, we shall first prove (2.2) when $N=N_{0}$ and $\left|\xi+i \tau N_{0}\right|=1$; then $\left|N_{0}\right|=1$ and

$$
\left|\xi+i \tau N_{0}\right|^{2}=\tau^{2}+\sum_{k=1}^{m} \xi_{k}^{2}=1 .
$$

When $\tau=0, P^{(\alpha)}(\xi) \neq 0$ for some $|\alpha|=s$ because $P$ has real characteristics of multiplicity at most $s$.

When $\tau \neq 0$ and $\left(\xi_{2}, \ldots, \xi_{n}\right) \neq 0$, then the polynomials $P^{(k)}\left(\xi+i \tau N_{0}\right), k=0, \ldots, r$ have no common zero, since $P$ has complex roots of multiplicity at most $r$. By Euler's theorem on homogeneous polynomials, the set of polynomials $P^{(\alpha)}\left(\xi+i \tau N_{0}\right)$, $|\alpha|=s$ and $P^{(k)}\left(\xi+i \tau N_{0}\right), k=s+1, \ldots, r$ have no common zero.

When $\tau \neq 0$ and $\left(\xi_{2}, \ldots, \xi_{n}\right)=0$, then the set of polynomials $P^{(\alpha)}\left(\xi+i \tau N_{0}\right)$, $|\alpha|=s$ becomes a set of monomials $c_{\alpha}\left(\xi_{1}-i \tau\right)^{m-s}$ with constant $c_{\alpha}$. For this set to have a common zero with $\xi_{1}^{2}+\tau^{2}=1$ would require all $c_{\alpha}=0$, but that would contradict the assumption on the multiplicity of the real roots of $P(\zeta)$.

Hence for $N=N_{0}$ and for all $(\xi, \tau)$ such that $\left|\xi+i \tau N_{0}\right|=1$, the sum on the right side of (2.2) is nonzero. That sum is a continuous positive function on the compact set $\tau^{2}+\sum_{k=1}^{n} \xi_{k}^{2}=1$, hence it has a minimum value $m_{0}>0$. Let $c=1 / m_{0}$, then (2.2) is proven for $\left|\xi+i \tau N_{0}\right|=1$. Since both sides of (2.2) are continuous functions in $N$, it follows that with a $C$ larger than $1 / m_{0}$, the proposition is true for all $N$ in some neighborhood $U$ of $N_{0}$ with $|N|=1$ and $|\xi+i \tau N|^{2}=\tau^{2}+\sum_{k=1}^{n} \xi_{k}^{2}=1$.

Next consider $\xi+i \tau N$ such that $(\xi, \tau) \neq 0$ and $N \in U$. Let

$$
\xi^{\prime}+i \tau^{\prime} N=(\xi+i \tau N) /|\xi+i \tau N| \text {. }
$$

Since the proposition is proven for $\left|\xi^{\prime}+i \tau^{\prime} N\right|=1$ and since $P(\zeta)$ is homogeneous of degree $m$, substituting into the inequality for $\xi^{\prime}+i \tau^{\prime} N$ yields (2.2) for $(\xi, \tau) \neq 0$ and $N \in U$. Finally if $(\xi, \tau)=(0,0)$, both sides of $(2.2)$ are either zero if $m>s$ or constant if $m=s$. Hence (2.2) is proven for $N \in U$.

Since $N$ and $\tau$ occur in (2.2) only as the product $\tau N$, it follows that (2.2) is valid for those $N \neq 0$ in the cone $V$, where $N \in V$ if $N /|N| \in U$. When $N=0,(2.2)$ holds because of the multiplicity of the real roots.

In the integrals to be studied, we shall use as a weight function $\exp (2 \tau \varphi(\delta, x))$, where $\tau$ is a parameter which will eventually be allowed to increase toward $+\infty$ 
and $\delta$ is a positive parameter which will be taken sufficiently small and then fixed. $\delta$ is the parameter introduced by Malgrange [4] and it enables us to include in (2.1) the terms of order $m-q$.

$$
\varphi \equiv \varphi(\delta, x)=\left(x_{1}-\delta\right)^{2}+\delta^{2}\left(x_{2}^{2}+\cdots+x_{n}^{2}\right)
$$

Notice that the surface $\varphi(\delta, x)=\varphi(\delta, 0)=\delta^{2}$ has a contact of the second order at the origin with the paraboloid $x_{1}=(\delta / 2)\left(x_{2}^{2}+\cdots+x_{n}^{2}\right)$, which apart from the origin lies in the set $x_{1}<\delta\left(x_{2}^{2}+\cdots+x_{n}^{2}\right)$. Let $U_{b}$ be a neighborhood of 0 such that when $x \in U_{\delta}$ then $\operatorname{grad} \varphi(\delta, x) \in V$ and $|\operatorname{grad} \varphi(\delta, x)-\operatorname{grad} \varphi(\delta, 0)|<\delta$, where $V$ is defined in Proposition 1. Using the methods of Hörmander [3], we shall now prove the basic Carleman type estimate.

THEOREM 1. Let $P(D)$ be a homogeneous operator of order $m$ with constant coefficients, with complex characteristics of multiplicity at most $r$, and with real characteristics of multiplicity at most $s$. Then when $u \in C_{0}^{\infty}\left(U_{b}\right),|\alpha| \leqq m-s, 1>\delta>0$, $\tau>\delta^{-2 s-2}, t=\max (r, s), p=2 s$ if $s \geqq r$ and $p=0$ if $s<r$,

$$
\delta^{p}\left(1+\delta^{2} \tau\right)^{m-|\alpha|-t} \tau^{m-|\alpha|} \int\left|D_{\alpha} u\right|^{2} e^{2 \tau \varphi} d x \leqq C \int|P(D) u|^{2} e^{2 \tau \Phi} d x .
$$

Proof. First we introduce a partition of unity, which is necessitated, as will be seen, by the fact that $\varphi$ is nonlinear. Let $\chi(x)=\chi\left(x_{1}, \ldots, x_{n}\right)$ be the characteristic function of the unit cube defined by $\left|x_{k}\right|<\frac{1}{2}, k=1, \ldots, n$. With a function $\Theta \geqq 0$ in $C_{0}^{\infty}\left(R^{n}\right)$ with integral 1 , we form the convolution

$$
\theta(x)=(\chi * \Theta)(x)=\int_{R^{n}} \chi(x-y) \Theta(y) d y .
$$

$\theta$ is a function in $C_{0}^{\infty}\left(R^{n}\right)$; moreover we may assume $\Theta$ is so chosen that the support of $\theta$ is contained in the cube $\left|x_{k}\right|<1, k=1,2, \ldots, n$. For fixed $x$ and $y, \Sigma_{g} \chi(x-g-y)$ $=1$ except on a set of measure zero, note $g=\left(g_{1}, \ldots, g_{n}\right)$ runs through all the integer coordinates. Hence $\sum_{g} \theta(x-g)=\int_{R^{n}} \Theta(y) d y=1$, and the functions $\theta(x-g)$ form a partition of unity. We shall use the partition of unity given by the functions $\theta\left(x_{1} \tau^{1 / 2}-g_{1}, x_{2} \delta \tau^{1 / 2}-g_{2}, \ldots, x_{n} \delta \tau^{1 / 2}-g_{n}\right)$ with $\tau, \delta>0$. Hence for $u \in C_{0}^{\infty}\left(U_{\delta}\right)$ $u=\sum, u_{g}$ where

$$
u_{g}(x)=\theta\left(x_{1} \tau^{1 / 2}-g_{1}, x_{2} \delta \tau^{1 / 2}-g_{2}, \ldots, x_{n} \delta \tau^{1 / 2}-g_{n}\right) u(x) .
$$

Notice that at most $2^{n}$ functions $u_{g}$ are different from zero at any one point.

Let $N_{\vartheta}=\operatorname{grad} \varphi\left(\delta, x_{g}\right)$ where $x_{q}=\left(g_{1} / \tau^{1 / 2}, g_{2} / \delta \tau^{1 / 2}, \ldots, g_{n} / \delta \tau^{1 / 2}\right)$. Let $x=x_{g} \in U_{\delta}$, hence $N_{g} \in V$ and Proposition 1 is valid for $N_{g}$. Let $\hat{u}_{g}\left(\xi+i \tau N_{g}\right)$ be the Fourier transform of $u_{g}(x) \exp \left(\left\langle x, \tau N_{g}\right\rangle\right),\left\langle x, N_{g}\right\rangle=\sum_{k=1}^{n} x_{k}\left(N_{g}\right)_{k}$. With $N=N_{g}$, we multiply (2.2) by $\left|\hat{u}_{g}\left(\xi+i \tau N_{g}\right)\right|^{2}$ and integrate,

$$
\begin{aligned}
& \int\left|\xi+i \tau N_{g}\right|^{2(m-s)}\left|\hat{u}_{g}\left(\xi+i \tau N_{g}\right)\right|^{2} d \xi \\
& \leqq C \int\left(\sum_{|\alpha|=s}\left|P^{(\alpha)}\left(\xi+i \tau N_{g}\right)\right|^{2}+\sum_{k=s+1}^{r}\left(\tau\left|N_{g}\right|\right)^{2(k-s)}\left|P^{(k)}\left(\xi+i \tau N_{g}\right)\right|^{2}\right) \\
& \cdot\left|\hat{u}_{g}\left(\xi+i \tau N_{g}\right)\right|^{2} d \xi .
\end{aligned}
$$


Let $\left|D^{m-s} u\right|^{2} \equiv \sum_{|\alpha|=m-s}\left|D_{\alpha} u\right|^{2}$. We shall use Parseval's formula; for $f \in C_{0}^{\infty}$

$$
\int_{R^{n}}\left|P^{(\alpha)}\left(\xi+i \tau N_{g}\right) \hat{f}\left(\xi+i \tau N_{g}\right)\right|^{2} d \xi=\int_{R^{n}}\left|\exp \left(\left\langle x, \tau N_{g}\right\rangle\right) P^{(\alpha)}(D) f(x)\right|^{2} d x .
$$

Using Parseval's formula on (2.6) yields

$$
\begin{aligned}
& \int\left|D^{m-s} u_{g}\right|^{2} \exp \left(2 \tau\left\langle x, N_{g}\right\rangle\right) d x \\
& \leqq C \int\left(\sum_{|\alpha|=s}\left|P^{(\alpha)}(D) u_{g}\right|^{2}+\sum_{k=s+1}^{r}\left(\tau\left|N_{g}\right|\right)^{2(k-s)}\left|P^{(k)}(D) u_{g}\right|^{2}\right) \\
& \cdot \exp \left(2 \tau\left\langle x, N_{g}\right\rangle\right) d x .
\end{aligned}
$$

Multiplying (2.7) by $\exp \left(2 \tau\left(\varphi\left(\delta, x_{g}\right)-\left\langle x_{g}, N_{g}\right\rangle\right)\right)$, we have

$$
\begin{aligned}
& \int\left|D^{m-s} u_{g}\right|^{2} \exp (2 \tau \psi) d x \\
& \quad \leqq C \int\left(\sum_{|\alpha|=s}\left|P^{(\alpha)}(D) u_{g}\right|^{2}+\sum_{k=s+1}^{r}\left(\tau\left|N_{g}\right|\right)^{2(k-s)}\left|P^{(k)}(D) u_{g}\right|^{2}\right) \exp (2 \tau \psi) d x,
\end{aligned}
$$

where $\psi=\varphi\left(\delta, x_{g}\right)+\left\langle x-x_{g}, N_{g}\right\rangle$.

LEMMA $1 . \varphi(\delta, x) \geqq \psi(\delta, x) \geqq \varphi(\delta, x)-n / \tau$ in the support of $u_{g}$.

Proof. $\varphi(\delta, x)=\psi(\delta, x)+\left(x_{1}-x_{g 1}\right)^{2}+\delta^{2} \sum_{k=2}^{n}\left(x_{k}-x_{g k}\right)^{2} \geqq \psi(\delta, x)$ by Taylor's formula. In the support of $u_{g}$, by $(2.5),\left(x_{1}-x_{g 1}\right)^{2}<1 / \tau$, and $\left(x_{k}-x_{g k}\right)^{2}<1 / \delta^{2} \tau$, $k=2, \ldots, n$; hence $\varphi(\delta, x) \leqq \psi(\delta, x)+n / \tau$.

Since $x_{g} \in U_{\delta},\left|N_{g}-\operatorname{grad} \varphi(\delta, 0)\right|<\delta$ and $\left|N_{g}\right|<3 \delta$. Using Lemma 1 and $\left|N_{g}\right|<3 \delta$ in (2.8)

$$
\begin{aligned}
& \int\left|D^{m-s} u_{g}\right|^{2} \exp (2 \tau \varphi) d x \\
& \quad \leqq C \int\left(\sum_{|\alpha|=s}\left|P^{(\alpha)}(D) u_{g}\right|^{2}+\sum_{k=s+1}^{r}(\tau \delta)^{2(k-s)}\left|P^{(k)}(D) u_{g}\right|^{2}\right) \exp (2 \tau \varphi) d x,
\end{aligned}
$$

where henceforth $C$ is a generic constant depending on $n, m$ and $P$ but independent of $\tau, \delta$ and $u$.

Since at most $2^{n}$ of the supports of the $u_{g}$ meet at any one point, we have in view of Cauchy's inequality

$$
\left|D^{m-s} u\right|^{2} \leqq 2^{n} \sum_{g}\left|D^{m-s} u_{g}\right|^{2}
$$

Let $\alpha^{\prime}$ denote the multi-index obtained by setting the first component of $\alpha$ equal to zero.

LeMma 2. $\sum_{g}\left|P^{(\alpha)}(D) u_{g}\right|^{2} \leqq C \sum_{\beta}\left|P^{(\alpha+\beta)}(D) u\right|^{2} \tau^{|\beta|} \delta^{2\left|\beta^{\prime}\right|}$. 
Proof. By (2.5) and Leibniz' formula

$$
P^{(\alpha)}(D) u_{g}=\sum_{\beta}\left(P^{(\alpha+\beta)}(D) u\right)\left(D_{\beta} \theta\right) \tau^{|\beta| / 2} \delta^{\left|\beta^{\prime}\right|}|| \beta \mid !
$$

Since $\theta \in C_{0}^{\infty}, \sum_{|\beta| \leqq m}\left|D_{\beta} \theta\right|^{2}$ is bounded. Also no more than $2^{n}$ functions $u_{g}$ are nonzero at any one point. Let $N(m)$ denote the number of terms in the set $P^{(\beta)}$, and let $C$ be a bound on $2^{n} N(m) \sum_{|\beta| \leqq m}\left|D_{\beta} \theta\right|^{2}$. By use of Cauchy's inequality from (2.11) we have $\sum_{g}\left|P^{(\alpha)}(D) u_{g}\right|^{2} \leqq C \sum_{\beta}\left|P^{(\alpha+\beta)}(D) u\right|^{2} \tau^{|\beta|} \delta^{2\left|\beta^{\prime}\right|}$.

Using Lemma 2 together with (2.10) on (2.9) yields

$$
\begin{aligned}
\int\left|D^{m-s} u\right|^{2} \exp (2 \tau \varphi) d x \leqq C \int \sum_{\beta} \tau^{|\beta|} \delta^{2\left|\beta^{\prime}\right|} \\
\cdot\left(\sum_{|\alpha|=s}\left|P^{(\alpha+\beta)}(D) u\right|^{2}+\sum_{k=s+1}^{r}(\tau \delta)^{2(k-s)}\left|P^{(k+\beta)}(D) u\right|^{2}\right) \exp (2 \tau \varphi) d x .
\end{aligned}
$$

Next we use a fundamental inequality, proven by Trèves [8]. If $Q(D)$ is a linear partial differential operator with constant coefficients, then for $u \in C_{0}^{\infty}$

$$
\left(t^{\alpha}\right)^{2} \int\left|Q^{(\alpha)}(D) u\right|^{2} e^{\eta} d x \leqq|\alpha| ! 2^{m-|\alpha|} \int|Q(D) u|^{2} e^{\eta} d x
$$

where $\eta=t_{1}^{2} x_{1}^{2}+\cdots+t_{n}^{2} x_{n}^{2}, t=\left(t_{1}, \ldots, t_{n}\right)$ and $t^{\alpha}=t_{1}^{\alpha_{1}} \cdots t_{n}^{\alpha_{n}}$. For the operator $P(D)$ in (2.1) we let $t_{1}^{2}=2 \tau, t_{2}^{2}=\cdots=t_{n}^{2}=2 \tau \delta^{2}$ and multiply $(2.13)$ by $\exp \left(2 \tau \delta^{2}\right)$ to obtain

$$
\tau^{|\alpha|} \delta^{2\left|\alpha^{\prime}\right|} \int\left|P^{(\alpha)}(D) u\right|^{2} \exp (2 \tau(\varphi+\mu)) d x \leqq C \int|P(D) u|^{2} \exp (2 \tau(\varphi+\mu)) d x
$$

where $\mu(\delta, x)=2 \delta x_{1}$. We apply (2.14) with $u e^{-\tau \mu}$ replacing $u$ and $P^{(\alpha)}\left(D+2 i \tau \delta N_{0}\right)$ replacing $P^{(\alpha)}(D)$. Since $P^{(\alpha)}\left(D+2 i \tau \delta N_{0}\right)\left(u e^{-\tau \mu}\right)=e^{-\tau \mu} P^{(\alpha)}(D) u$ we obtain

$$
\tau^{|\alpha|} \delta^{2\left|\alpha^{\prime}\right|} \int\left|P^{(\alpha)}(D) u\right|^{2} e^{2 \tau \varphi} d x \leqq C \int|P(D) u|^{2} e^{2 \tau \varphi} d x .
$$

Using (2.15) to estimate two typical terms on the right side of (2.12)

$$
\tau^{|\beta|} \delta^{2\left|\beta^{\prime}\right|} \int\left|P^{(\alpha+\beta)}(D) u\right|^{2} e^{2 \tau \varphi} d x \leqq C\left(\delta^{2} \tau\right)^{-s} \int|P(D) u|^{2} e^{2 \tau \varphi} d x
$$

since $\delta<1$ and $|\alpha|=s$, and

$$
\begin{aligned}
\tau^{|\beta|} \delta^{2\left|\beta^{\prime}\right|}(\tau \delta)^{2(k-s)} \int\left|P^{(k+\beta)}(D) u\right|^{2} e^{2 \tau \varphi} & d x \\
& \leqq C\left(\delta^{2} \tau\right)^{k}(\delta \tau)^{-2 s} \int|P(D) u|^{2} e^{2 \tau \varphi} d x .
\end{aligned}
$$

If $s \geqq r$, using (2.16) in (2.12) yields

$$
\int\left|D^{m-s} u\right|^{2} e^{2 \tau \varphi} d x \leqq C\left(\delta^{2} \tau\right)^{-s} \int|P(D) u|^{2} e^{2 \tau \varphi} d x .
$$


If $r>s$, then by choosing $\tau>\delta^{-2 s-2}$ and using (2.16) and (2.17) in (2.12)

$$
\int\left|D^{m-s} u\right|^{2} e^{2 \tau \varphi} d x \leqq C \tau^{-s}\left(1+\tau \delta^{2}\right)^{r-s} \int|P(D) u|^{2} e^{2 \tau \varphi} d x
$$

All lower order derivatives are estimated by integrals of $\left|D^{m-s} u\right|^{2}$.

LeMma 3. If $v \in C_{0}^{\infty}\left(U_{\delta}\right)$ then

$$
\tau\left(1+\delta^{2} \tau\right) \int|v|^{2} e^{2 \tau \varphi} d x \leqq \int\left|D_{1} v\right|^{2} e^{2 \tau \varphi} d x
$$

Proof. Let $w=v e^{\tau \varphi}$, then

$$
\begin{aligned}
\int\left|D_{1} v\right|^{2} e^{2 \tau \omega} d x= & \int\left|\partial w / \partial x_{1}\right|^{2} d x+4 \tau^{2} \int\left(x_{1}-\delta\right)^{2}|w|^{2} d x \\
& -2 \tau \int\left(x_{1}-\delta\right) \partial|w|^{2} / \partial x_{1} d x
\end{aligned}
$$

Using integration by parts $-2 \tau \int\left(x_{1}-\delta\right) \partial|w|^{2} / \partial x_{1} d x=2 \tau \int|w|^{2} d x$.

$$
\int\left|D_{1} v\right|^{2} e^{2 \tau \omega} d x \geqq \int\left(\tau+4 \tau^{2}\left(x_{1}-\delta\right)^{2}\right)|w|^{2} d x .
$$

For $x \in U_{\delta}, 4\left(x_{1}-\delta\right)^{2}>\delta^{2}$.

Using Lemma 3 repeatedly with $v=D_{\alpha} u$ and $|\alpha| \leqq m-s$

$$
\left(\tau+\delta^{2} \tau^{2}\right)^{m-|\alpha|-s} \int\left|D_{\alpha} u\right|^{2} e^{2 \tau \omega} d x \leqq \int\left|D^{m-s} u\right|^{2} e^{2 \tau \Phi} d x
$$

(2.18) and (2.20) yield

$$
\delta^{2 s}\left(1+\delta^{2} \tau\right)^{m-|\alpha|-s} \tau^{m-|\alpha|} \int\left|D_{\alpha} u\right|^{2} e^{2 \tau \varphi} d x \leqq C \int|P(D) u|^{2} e^{2 \tau \varphi} d x .
$$

(2.19) and (2.20) yield

$$
\left(1+\delta^{2} \tau\right)^{m-|\alpha|-r} \tau^{m-|\alpha|} \int\left|D_{\alpha} u\right|^{2} e^{2 \tau \Phi} d x \leqq C \int|P(D) u|^{2} e^{2 \tau \Phi} d x
$$

and the theorem is proven. $C$ depends on $m, n$ and $P$ but is independent of $u, \tau$ and $\delta$.

We shall now use Theorem 1 to prove uniqueness of solutions to the Cauchy problem for (2.1).

THEOREM 2. Let $P(D)$ satisfy the hypothesis of Theorem 1 and let $u \in C^{m}$ be a solution of (2.1) which vanishes for $x_{1} \leqq \varepsilon\left(x_{2}^{2}+\cdots+x_{n}^{2}\right), \varepsilon>0$, when $x$ is in a neighborhood of the origin. Then $u$ vanishes in a neighborhood of the origin.

Proof. Let $U_{\delta}$ be a neighborhood of 0 such that (2.4) is valid and $U_{\delta}$ belongs to 
the neighborhood given in the hypothesis of this theorem. Let $\chi \in C_{0}^{\infty}\left(U_{\delta}\right)$ such that $\chi=1$ in a neighborhood $U^{\prime}$ of 0 and let $v=\chi u$. Thus in $U^{\prime}$

$$
|P v| \leqq K \sum_{|\alpha| \leq m-q}\left|D_{\alpha} v\right|
$$

When $x \in U_{\delta}$ and $x$ is also in the support of $v$ then $\varepsilon\left(x_{2}^{2}+\cdots+x_{n}^{2}\right) \leqq x_{1} \leqq \delta$. Let $\delta \leqq \varepsilon$, then $\varphi(\delta, x)<\delta^{2}$ except when $x=0$. If in addition $x \in C U^{\prime}$, the complement of $U^{\prime}$, then $\varphi \leqq \delta^{2}-L$ where $0<L<\delta^{2} / 2$. Also let $U^{\prime \prime} \subset U^{\prime}$ be a neighborhood of the origin where $\varphi>\delta^{2}-L / 2$; note $\varphi(\delta, 0)=\delta^{2}$.

Since $C_{0}^{\infty}\left(U_{\delta}\right)$ is dense in $L_{2}\left(U_{\delta}\right)$ we may apply (2.4) to $v$ :

$$
\begin{aligned}
\sum_{|\alpha| \leq m-q} \delta^{p} \tau^{m-|\alpha|}\left(1+\delta^{2} \tau\right)^{m-|\alpha|-t} \int\left|D_{\alpha} v\right|^{2} e^{2 \tau \varphi} d x \\
\leqq C \int|P(D) v|^{2} e^{2 \tau \varphi} d x \\
\quad \leqq K C \int_{U^{\prime}} \sum_{|\alpha| \leq m-q}\left|D_{\alpha} v\right|^{2} e^{2 \tau \varphi} d x+C \int_{C U}|P(D) v|^{2} e^{2 \tau \varphi} d x
\end{aligned}
$$

where (2.21) is used to obtain the second inequality. When $r$ is an even number, $s \leqq r / 2$ and $|\alpha|=m-r / 2$, then as $\tau \rightarrow+\infty, \delta^{p} \tau^{m-|\alpha|}\left(1+\delta^{2} \tau\right)^{m-|\alpha|-r} \rightarrow \delta^{-r}$; choose $\delta^{-1} \gg K C$ and then fix $\delta$. In all other cases as $\tau \rightarrow+\infty$, then $\delta^{p} \tau^{m-|\alpha|}\left(1+\delta^{2} \tau\right)^{m-|\alpha|-t}$ $\rightarrow+\infty$. Thus in all cases for $\delta$ sufficiently small and then fixed and for $\tau$ sufficiently large we may move the terms involving $D_{\alpha} v$ on the right side of (2.22) to the left side to obtain with a larger $C$

$$
\sum_{|\alpha| \leqq m-q} \delta^{p} \tau^{m-|\alpha|}\left(1+\delta^{2} \tau\right)^{m-|\alpha|-t} \int\left|D_{\alpha} v\right|^{2} e^{2 \tau \varphi} d x \leqq C \int_{C U^{\prime}}|P(D) v|^{2} e^{2 \tau \varphi} d x .
$$

Dropping all terms on the left side except the one for $|\alpha|=0$ and restricting the integration for that term to $U^{\prime \prime}$

$$
\int_{U^{*}}|v|^{2} e^{2 \tau \varphi} d x \leqq C \int_{C U^{\prime}}|P(D) v|^{2} e^{2 \tau \varphi} d x .
$$

Using in (2.23) the fact that $\varphi \leqq \delta^{2}-L$ when $x \in C U^{\prime}$ and $x$ is in the support of $v$ and $\varphi>\delta^{2}-L / 2$ when $x \in U^{\prime \prime}$, we have

$$
\int_{U^{\prime \prime}}|v|^{2} d x \leqq C e^{-\tau L} \int_{C U^{\prime}}|P(D) v|^{2} d x
$$

When $\tau \rightarrow+\infty$ it follows that the inequality can hold only if $u=v \equiv 0$ in $U^{\prime \prime}$, and the theorem is proven.

Hence solutions of the Cauchy problem for (2.1) are unique across those convex surfaces $S$ which at any point $\bar{x}$ of $S$ admit coordinates such that in a neighborhood of $\bar{x}, S$ does not lie below $x_{1}=\varepsilon\left(x_{2}^{2}+\cdots+x_{n}^{2}\right)$, for some $\varepsilon>0$. 
3. Parabolic equations with constant leading coefficients. For this section only we change the notation slightly. Here $\Omega$ denotes an open set in real $n+1$ dimensional Euclidean space $R^{n+1}, n \geqq 1 . x=\left(t, x_{1}, \ldots, x_{n}\right) \in \Omega$.

$$
\begin{array}{rlrl}
\alpha=\left(\alpha_{t}, \alpha_{1}, \ldots, \alpha_{n}\right) . & \alpha^{\prime}=\left(0, \alpha_{1}, \ldots, \alpha_{n}\right) . & |\alpha|=\alpha_{t}+\sum_{k=1}^{n} \alpha_{k} . \\
\xi=\left(\xi_{t}, \xi_{1}, \ldots, \xi_{n}\right) \in R^{n+1} . & \xi^{\alpha}=\xi_{t}^{\alpha_{t}} \xi_{1}^{\alpha_{1}} \ldots \xi_{n}^{\alpha_{n} .} & \xi^{\prime}=\left(0, \xi_{1}, \ldots, \xi_{n}\right) . \\
\zeta=\left(\zeta_{t}, \zeta_{1}, \ldots, \zeta_{n}\right) \in C^{n+1} . & D_{t}=-i \partial / \partial t . & D=\left(D_{t}, D_{1}, \ldots, D_{n}\right) . \\
D_{\alpha}=D_{t}^{\alpha_{t}} D_{1}^{\alpha_{1}} \ldots D_{n}^{\alpha_{n} .} &
\end{array}
$$

An operator $P(D)$ of order $m$ with constant coefficients will be called parabolic if it has the form

$$
P(D)=\partial / \partial t-P_{e}(D)
$$

$P_{e}(D)$ is a polynomial in $D_{k}$ for $k=1, \ldots, n$. For $n \geqq 2, P_{e}(D)$ is a homogeneous elliptic operator of order $m$ with characteristics of multiplicity at most $r$ when considered as an operator in the variables $x_{1}, \ldots, x_{n}$. For $n=1, P_{e}(D)=c D_{1}^{m}$ with $c$ a nonzero constant, $m$ even and $i^{m} c>0$.

We shall consider two problems. First we study the solutions of the differential inequality

$$
|P(D) u| \leqq K \sum_{\left|\alpha^{\prime}\right| \leqq m-q}\left|D_{\alpha^{\prime}} u\right|,
$$

where $P(D)$ is the parabolic operator (3.1). $q=[(r+1) / 2]$.

Proposition 2. Let $N_{0}=(0,-1,0, \ldots, 0), \xi \in R^{n+1}, \tau \in R$, then there is an open cone $V \subset R^{n+1}$ with $N_{0} \in V$ such that for $N \in V, N=\left(N_{t}, N_{1}, \ldots, N_{n}\right), N^{\prime}=$ $\left(0, N_{1}, \ldots, N_{n}\right)$ we have

$$
\begin{aligned}
& \left|\xi^{\prime}+i \tau N^{\prime}\right|^{2(m-1)} \\
& \quad \leqq C\left(\sum_{\left|\alpha^{\prime}\right|=1}\left|P^{\left(\alpha^{\prime}\right)}(\xi+i \tau N)\right|^{2}+\sum_{k=2}^{r}(\tau|N|)^{2(k-1)}\left|P^{(k)}(\xi+i \tau N)\right|^{2}\right) .
\end{aligned}
$$

Proof. From (3.1) we have $P^{\left(\alpha^{\prime}\right)}(\zeta)=-P_{e}^{\left(\alpha^{\prime}\right)}(\zeta)$ for $\left|\alpha^{\prime}\right| \geqq 1$. Since both sides of (3.3) are independent of $\xi_{t}$, we prove (3.3) with $\xi_{t}=0$. The remainder of the proof is the same as for Proposition 1, but here we replace $P$ with $P_{e}$ and use the elliptic and homogeneous properties of $P_{e}$. Note if $r=1$ then there is no second sum in (3.3).

Using Proposition 2 a Carleman type estimate analogous to Theorem 1 can be proven for the parabolic operator (3.1) with $\alpha^{\prime}$ replacing $\alpha, s=1$ and $\varphi(\delta, x)$ $=\left(x_{1}-\delta\right)^{2}+\delta^{2}\left(x_{2}^{2}+\cdots+x_{n}^{2}+t^{2}\right)$ in (2.4). Using that estimate and following the proof of Theorem 2, it follows that solutions $u \in C^{m}$ to (3.2) which vanish for $x_{1} \leqq \varepsilon\left(x_{2}^{2}+\cdots+x_{n}^{2}+t^{2}\right), \varepsilon>0$, when $x$ is in a neighborhood of the origin, do in fact vanish in a neighborhood of the origin. 
Next we study uniqueness of the Cauchy problem for parabolic operators across the surface $t=\varepsilon\left(x_{1}^{2}+\cdots+x_{n}^{2}\right), \varepsilon>0$. We study solutions of the differential inequality (3.2) but with $q=1$, hence now (3.2) is independent of the multiplicity of the complex characteristics of $P_{e}(D)$.

Proposition 3. Let $N_{0}=(-1,0, \ldots, 0) \in R^{n+1}, \xi \in R^{n+1}, \tau \in R$, then there is an open cone $V \subset R^{n+1}$ with $N_{0} \in V$ such that for $N \in V, N=\left(N_{t}, N_{1}, \ldots, N_{n}\right)$, $N^{\prime}=\left(0, N_{1}, \ldots, N_{n}\right)$ we have

$$
\left|\xi^{\prime}+i \tau N^{\prime}\right|^{2(m-1)} \leqq C\left(\sum_{\left|\alpha^{\prime}\right|=1}\left|P^{\left(\alpha^{\prime}\right)}(\xi+i \tau N)\right|^{2}\right) .
$$

Proof. Since both sides of (3.4) are independent of $\xi_{t}$, we prove (3.4) with $\xi_{t}=0$. We first prove (3.4) when $N=N_{0}$ and $\left|\xi+i \tau N_{0}\right|^{2}=\tau^{2}+\sum_{k=1}^{n} \xi_{k}^{2}=1$. Then

$$
\left|\xi^{\prime}+i \tau N^{\prime}\right|^{2(m-1)}=\left|\xi^{\prime}+i \tau N_{0}^{\prime}\right|^{2(m-1)}=\left|\xi^{\prime}\right|^{2(m-1)}
$$

and from (3.1) for $\left|\alpha^{\prime}\right|=1$

$$
P^{\left(\alpha^{\prime}\right)}(\xi+i \tau N)=-P_{e}^{\left(\alpha^{\prime}\right)}\left(\xi+i \tau N_{0}\right)=-P_{e}^{\left(\alpha^{\prime}\right)}(\xi) .
$$

When $\tau=0,\left|\xi+i \tau N_{0}\right|=|\xi|=1$, hence $P_{e}^{\left(\alpha^{\prime}\right)}(\xi) \neq 0$ for some $\left|\alpha^{\prime}\right|=1$, by the use of Euler's theorem on homogeneous polynomials and the fact that $P_{e}(\xi)$ is elliptic in $\xi^{\prime}$. Therefore the sum $\sum_{\left|\alpha^{\prime}\right|=1}\left|P^{(\alpha)}(\xi)\right|^{2}$ is a continuous positive function on the compact set $|\xi|=1$, hence it has a minimum value $m_{0}>0$. Let $C=1 / m_{0}$, then

$$
\left|\xi^{\prime}\right|^{2(m-1)}=1 \leqq C \sum_{\left|\alpha^{\prime}\right|=1}\left|P^{\left(\alpha^{\prime}\right)}(\xi)\right|^{2}, \text { for }|\xi|=1 .
$$

Since both sides of (3.7) are homogeneous of order $2(m-1)$ in $\xi_{k}, k=1, \ldots, n$, (3.7) is valid for all $\xi$. Using (3.5) and (3.6) in (3.7) yields

$$
\left|\xi^{\prime}+i \tau N_{0}^{\prime}\right|^{2(m-1)} \leqq C \sum_{\left|\alpha^{\prime}\right|=1}\left|P^{\left(\alpha^{\prime}\right)}\left(\xi+i \tau N_{0}\right)\right|^{2}
$$

and (3.4) is proven when $N=N_{0}$ and $\left|\xi+i \tau N_{0}\right|=1$.

Since both sides of (3.4) are continuous functions in $N$, it follows that with a larger $C$ than $1 / m_{0}$, the proposition is true for all $N$ in some neighborhood of $N_{0}$ with $|N|=1$ and $|\xi+i \tau N|^{2}=\tau^{2}+\sum_{k=1}^{n} \xi_{k}^{2}=1$. The remainder of the proof is exactly the same as that of Proposition 1 .

So we see that when $N_{0}$ points in the $t$ direction of $R^{n+1}$, then we can choose $V$ "narrow" enough so that we miss the complex roots of $P_{e}(\zeta)$. Now we shall prove a Carleman type estimate for $P$ similar to $(2.4)$, but here we set

$$
\varphi(\delta, x)=(t-\delta)^{2}+\delta^{2}\left(x_{1}^{2}+\cdots+x_{n}^{2}\right) .
$$

Let $U_{\delta}$ be defined as in $\S 2$ except that here we use (3.8) and the $V$ defined by Proposition 3. 
THEOREM 3. Let $P(D)$ be the parabolic operator defined by (3.1). Then when $u \in C_{0}^{\infty}\left(U_{\delta}\right),\left|\alpha^{\prime}\right| \leqq m-1, \tau>0$ and $1>\delta>0$

$$
\left(\tau \delta^{2}\right)^{m-\left|\alpha^{\prime}\right|} \int\left|D_{\alpha^{\prime}} u\right|^{2} e^{2 \tau \varphi} d x \leqq C \int|P(D) u|^{2} e^{2 \tau \varphi} d x .
$$

Proof. The proof is similar to that of Theorem 1 so we shall only indicate the changes. Let $\left|D^{m-1} u\right|^{2} \equiv \sum_{\left|\alpha^{\prime}\right|=m-1}\left|D_{\alpha^{\prime}} u\right|^{2}$. The proof proceeds as before but now instead of Proposition 1 we use Proposition 3 to obtain the basic estimate (similar to $(2.18)$ )

$$
\tau \delta^{2} \int\left|D^{m-1} u\right|^{2} e^{2 \tau \Phi} d x \leqq C \int|P(D) u|^{2} e^{2 \tau \Phi} d x .
$$

Lemma 3 is not useful here because its use is dependent upon the basic estimate estimating derivatives in the $N_{0}$ direction, which (3.10) does not do. However when $\varphi$ is defined by (3.8) we have

LEMMA 4. If $v \in C_{0}^{\infty}\left(U_{\delta}\right)$ and $D_{1}=-i \partial / \partial x_{1}$

$$
\tau \delta^{2} \int|v|^{2} e^{2 \tau \varphi} d x \leqq \int\left|D_{1} v\right|^{2} e^{2 \tau \varphi} d x .
$$

The proof is similar to that of Lemma 3 . Using Lemma 4 repeatedly together with (3.10) yields (3.9).

Using (3.9) and following the proof of Theorem 2, it follows that solutions $u \in C^{m}$ to (3.2) which vanish for $t \leqq \varepsilon\left(x_{1}^{2}+\cdots+x_{n}^{2}\right), \varepsilon>0$, when $x$ is in a neighborhood of the origin, do in fact vanish in a neighborhood of the origin.

4. Elliptic equations with variable leading coefficients. We shall study the solution of the differential inequality

$$
|P(x, D) u| \leqq K \sum_{|\alpha| \leqq m-q}\left|D_{\alpha} u\right|,
$$

where $P(x, D)$ is an elliptic operator of order $m$ with characteristics of multiplicity at most $r$ and $q=[(r+1) / 2] . u \in C^{m}$ is a solution of (4.1) which vanishes for $x_{1} \leqq x_{2}^{2}+\cdots+x_{n}^{2}$ when $x$ is in a neighborhood of the origin. $P(x, D)$ also has the following form.

$$
P(x, D)=P_{1}(x, D) P_{2}(x, D) \cdots P_{r}(x, D) .
$$

Each of the factors $P_{k}(x, D), k=1, \ldots, r$ in the product (4.2) satisfies the following conditions.

$$
P_{k}(x, D)=\sum_{|\alpha| \leqq m_{k}} a_{\alpha}^{k}(x) D_{\alpha}
$$

is an operator of order $m_{k}$ with coefficients $a_{\alpha}^{k}(x)$ such that for $k=2, \ldots, r, a_{\alpha}^{k}(x)$ have Lipschitz continuous derivatives of order $\left(\sum_{l=1}^{k-1} m_{l}\right)-1$, and for $k=1, a_{\alpha}^{k}(x)$ 
are Lipschitz continuous for $|\alpha|=m_{1}$ and $L_{\infty}$ for $|\alpha|<m_{1}$. Let $P_{k, m}(x, D) \equiv$ $\sum_{|\alpha|=m_{k}} a_{\alpha}^{k}(x) D_{\alpha}$ denote the principal part of $P_{k}(x, D)$; we assume $P_{k}(x, D)$ is elliptic at the origin, i.e.,

$$
P_{k, m}(0, \xi) \neq 0 \quad \text { for } 0 \neq \xi \in R^{n} .
$$

Finally we assume that $P_{k}(x, D)$ has simple characteristics at the origin, i.e.,

$$
P_{k, m}\left(0, \zeta_{1}, \xi_{2}, \ldots, \xi_{n}\right)=0
$$

has distinct roots for $\zeta_{1}$ complex and $0 \neq\left(\xi_{2}, \ldots, \xi_{n}\right) \in R^{n-1}$.

Each $P_{k, m}(x, \zeta)$ satisfies an inequality analogous to $(2.2)$ for operators with constant coefficients. Let

$$
P^{(l)}(x, \zeta) \equiv \partial P(x, \zeta) / \partial \zeta_{l}, \quad l=1, \ldots, n .
$$

Proposition 4. Let $N_{0}=(-1,0, \ldots, 0), \xi \in R^{n}, \tau \in R$; then there is a compact neighborhood $U^{k}$ of the origin and an open cone $V^{k} \subset R^{n}$ with $N_{0} \in V^{k}$ such that for $x \in U^{k}$ and $N \in V^{k}$

$$
\begin{gathered}
|\xi+i \tau N|^{2 m_{k}} \leqq C\left(\left|P_{k, m}(x, \xi+i \tau N)\right|^{2}+(\tau|N|)^{2}\left|P_{k, m}^{(1)}(x, \xi+i \tau N)\right|^{2}\right), \\
|\xi+i \tau N|^{2\left(m_{k}-1\right)} \leqq C\left(\sum_{l=1}^{n}\left|P_{k, m}^{(l)}(x, \xi+i \tau N)\right|^{2}\right) .
\end{gathered}
$$

Proof. The proof of (4.6) for $N=N_{0}, x=0$ and $\left|\xi+i \tau N_{0}\right|=1$ is exactly the same as that for Proposition 1. The extension to a compact neighborhood $U^{k}$ of the origin follows from the fact that the right side of (4.6) is a continuous function of $x$. The remainder of the proof of (4.6) is exactly the same as for (2.2). Using Euler's theorem on homogeneous polynomials, a similar proof yields (4.7), and the proposition is proven.

Just as in $\S 2$, we shall use $e^{2 \tau \omega}$ as a weight function in the integrals, but in this section

$$
\varphi=\varphi(\delta, x)=\left(x_{1}-\delta\right)^{2}+\delta\left(x_{2}^{2}+\cdots+x_{n}^{2}\right) .
$$

Let $U_{\delta}^{k} \subset U^{k}$ be a neighborhood of 0 such that when $x \in U_{\delta}^{k}$ then $\operatorname{grad} \varphi(\delta, x) \in V^{k}$ and $|\operatorname{grad} \varphi(\delta, x)-\operatorname{grad} \varphi(\delta, 0)|<\delta$, where $V^{k}$ is defined in Proposition 4. Finally let $U_{\delta}=\bigcap_{k=1}^{r} U_{\delta}^{k}$; we shall prove the basic Carleman-type estimate.

THEOREM 4. Let $P(x, D)$ be an elliptic operator of order $m$ with characteristics of multiplicity at most $r$ and let $P(x, D)$ satisfy (4.2). Then when $u \in C_{0}^{\infty}\left(U_{\delta}\right),|\alpha| \leqq m$, $\tau>0, \delta_{0}>\delta>0, \tau \delta>M$, where $M$ and $\delta_{0}$ are constants

$$
\left(1+\delta^{2} \tau\right)^{m-|\alpha|-r} \tau^{m-|\alpha|} \int\left|D_{\alpha} u\right|^{2} e^{2 \tau \varphi} d x \leqq C \int|P(x, D) u|^{2} e^{2 \tau \varphi} d x .
$$

Note that (4.9) is similar in form to (2.4). 
Proof. By induction on $r$. First we simplify the notation. Let $\nu \equiv 1+\delta^{\mathbf{2}} \tau$, $\|u\|_{\alpha}^{2} \equiv \int\left|D_{\alpha} u\right|^{2} e^{2 \tau \omega} d x$ and $\|u\|_{p}^{2} \equiv \int|P(x, D) u|^{2} e^{2 \tau \omega} d x$. $\|u\|_{p, k}^{2}$ and $\|u\|_{p, k, m}^{2}$ are the integral $\|u\|_{p}^{2}$ with $P_{k}(x, D)$ and $P_{k, m}(x, D)$ respectively replacing $P(x, D)$.

First let $r=1$; Hörmander [3(b)] proved (4.9) for $P(x, D)=P_{1, m}(x, D)$, the principal part of $P_{1}(x, D)$. Hence

$$
\sum_{|\alpha| \leqq m_{1}} \nu^{m_{1}-|\alpha|-1} \tau^{m_{1}-|\alpha|}\|u\|_{\alpha}^{2} \leqq C\|u\|_{p, 1, m}^{2},
$$

where $C$ denotes a generic constant which depends on $n, m$ and $P$ but is independent of $\tau, \delta$ and $u$. By using Cauchy's inequality on (4.3) with $k=1$ and the fact that the $a_{\alpha}^{1}(x) \in L_{\infty}$

$$
\left|P_{1, m}(x, D) u\right|^{2} \leqq 2\left|P_{1}(x, D) u\right|^{2}+C \sum_{|\alpha|<m_{1}}\left|D_{\alpha} u\right|^{2} .
$$

(4.10) and (4.11) yield

$$
\sum_{|\alpha| \leqq m_{1}} \nu^{m_{1}-|\alpha|-1} \tau^{m_{1}-|\alpha|}\|u\|_{\alpha}^{2} \leqq C\|u\|_{p, 1}^{2}+C \sum_{|\alpha|<m_{1}}\|u\|_{\alpha}^{2} .
$$

For $\tau>2 C$ the second term on the right side can be moved to the left to obtain (4.9) when $r=1$.

Next assume (4.9) is valid for $r-1$; we shall prove that (4.9) is then valid for $r$. Let $m^{\prime} \equiv \sum_{k=1}^{r-1} m_{k}$ and $P^{\prime}(x, D)=P_{1}(x, D) \cdots P_{r-1}(x, D)$. From the assumption that (4.9) is valid for $r-1$ we have

$$
\sum_{|\alpha| \leqq m^{\prime}} \nu^{m^{\prime}-|\alpha|-r+1} \tau^{m^{\prime}-|\alpha|}\|u\|_{\alpha}^{2} \leqq C\|u\|_{D^{\prime}}^{2}
$$

Next we replace $u$ in (4.13) with $P_{r}(x, D) u$; that the resulting inequality is valid follows from approximation since $C_{0}^{\infty}$ is dense in $L_{2}$ and we obtain

$$
\sum_{|\alpha| \leqq m^{\prime}} \nu^{m^{\prime}-|\alpha|-r+1} \tau^{m^{\prime}-|\alpha|}\left\|D_{\alpha} P_{r}(x, D) u\right\|_{0}^{2} \leqq C\|u\|_{p}^{2},
$$

where $\|u\|_{0}=\|u\|_{\alpha}$ with $|\alpha|=0$.

Now

$$
D_{\alpha} P_{r}(x, D) u=P_{r, m}(x, D) D_{\alpha} u+\sum_{|\beta|<m_{r}+|\alpha|} c_{\beta}^{r}(x) D_{\beta} u,
$$

where the $c_{\beta}^{\tau}(x)$ are bounded and measurable in the support of $u$. Hence by using Cauchy's inequality on (4.15) and the result in (4.14)

$$
\begin{aligned}
\sum_{|\alpha| \leqq m^{\prime}} \nu^{m^{\prime}-|\alpha|-r+1} \tau^{m^{\prime}-|\alpha|}\left\|P_{r, m}(x, D) D_{\alpha} u\right\|_{0}^{2} & \\
& \leqq C\|u\|_{p}^{2}+C \sum_{|\alpha| \leqq m^{\prime}} \nu^{m^{\prime}-|\alpha|-r+1} \tau^{m^{\prime}-|\alpha|} \sum_{|\beta|<m_{r}+|\alpha|}\|u\|_{\beta}^{2} .
\end{aligned}
$$$$
|\beta|<m_{r}+|\alpha| \leqq m_{r}+m^{\prime}=m \text {. }
$$ 
From (4.17) $m^{\prime}-|\alpha| \leqq m-|\beta|-1$, let $\tau>1$ and since $\nu>1$

$$
\tau^{m^{\prime}-|\alpha|} \leqq \tau^{m-|\beta|-1} \text { and } \nu^{m^{\prime}-|\alpha|-r+1} \leqq \nu^{m-|\beta|-r} .
$$

Using (4.17) and (4.18) in (4.16) yields

$$
\begin{aligned}
\sum_{|\alpha| \leqq m^{\prime}} \nu^{m^{\prime}-|\alpha|-r+1} \tau^{m^{\prime}-|\alpha|} \| P_{r, m}( & , D) D_{\alpha} u \|_{0}^{2} \\
& \leqq C\|u\|_{p}^{2}+C \sum_{|\beta|<m} \nu^{m-|\beta|-r} \tau^{m-|\beta|-1}\|u\|_{B}^{2} .
\end{aligned}
$$

Since $D_{\alpha} u \in C_{0}^{\infty}\left(U_{\delta}\right)$ and since (4.9) has been proven for $P(x, D)$ with simple characteristics, i.e., $r=1$, we may use (4.9) for $P(x, D)=P_{r, m}(x, D)$

$$
\sum_{|\beta| \leqq m_{r}} \nu^{m_{r}-|B|-1} \tau^{m_{r}}-|B|\left\|D_{B} D_{\alpha} u\right\|_{0}^{2} \leqq C\left\|P_{r, m}(x, D) D_{\alpha} u\right\|_{0}^{2}
$$

Using (4.20) in (4.19), with $D_{\beta} D_{\alpha}=D_{\beta+\alpha}$, and $|\beta+\alpha|=|\beta|+|\alpha|$

$$
\sum_{|\alpha| \leqq m} \nu^{m-|\alpha|-r} \tau^{m-|\alpha|}\|u\|_{\alpha}^{2} \leqq C\|u\|_{p}^{2}+C \sum_{|\alpha|<m} \nu^{m-|\alpha|-r} \tau^{m-|\alpha|-1}\|u\|_{\alpha}^{2}
$$

Since the power of $\tau$ is one higher on the left side of (4.21), we take $\tau>2 C$ and move the second term on the right to the left to obtain

$$
\sum_{|\alpha| \leqq m} \nu^{m-|\alpha|-r} \tau^{m-|\alpha|}\|u\|_{\alpha}^{2} \leqq C\|u\|_{p}^{2}
$$

So (4.9) is proven for $r$ and the induction is completed.

We now use Theorem 4 to prove uniqueness of solutions to the Cauchy problem for (4.1).

THEOREM 5. Let $P(x, D)$ satisfy the hypothesis of Theorem 4 and let $u \in C^{m}$ be a solution of (4.1) which vanishes for $x_{1} \leqq x_{2}^{2}+\cdots+x_{n}^{2}$ when $x$ is in a neighborhood of the origin. Then $u$ vanishes in a neighborhood of the origin.

Proof. The proof is exactly the same as the proof of Theorem 2 with $\varepsilon=1$, but here we use the estimate (4.9) instead of (2.4).

Let $S$ be an arbitrary surface of class $C^{m}$ and let $\bar{x}$ be a point on $S$. By a change of coordinates the surface $S$ can be made to coincide with the paraboloid $x_{1}=x_{2}^{2}+$ $\cdots+x_{n}^{2}$ in a neighborhood of $\bar{x}$ with $\bar{x}$ at the origin of the new coordinates. The class of differential inequalities considered in (4.1) is invariant under the change of coordinates and hence Theorem 5 implies uniqueness of solutions to the Cauchy problem for (4.1) across arbitrary surfaces.

The results of $\$ 2$ and this section can be combined. Consider the differential inequality

$$
|P(x, D) u| \leqq K \sum_{|\alpha| \leqq m-p}\left|D_{\alpha} u\right|,
$$

where $P(x, D)$ is an operator of order $m$ with complex characteristics of multiplicity 
at most $r$ and real characteristics of multiplicity at most $s, q=\max (s,[(r+1) / 2])$. $P(x, D)$ also has the following form

$$
P(x, D)=P_{1}(x, D) \cdots P_{h}(x, D),
$$

where each of the factors in (4.23) is the type of operator considered in either (2.1) or (4.1). By the method used to prove Theorem 4, a Carleman type estimate similar to (2.4) can be proven for (4.23). But now $\varphi(\delta, x)=\left(x_{1}-\delta\right)^{2}+\delta^{b}\left(x_{2}^{2}+\cdots+x_{n}^{2}\right)$, $1<b<2$. That estimate then implies that solutions $u \in C^{m}$ of (4.22), which vanish for $x_{1} \leqq \varepsilon\left(x_{2}^{2}+\cdots+x_{n}^{2}\right), \varepsilon>0$, when $x$ is in a neighborhood of the origin, do in fact vanish in a neighborhood of the origin.

5. Comparison of the results in $\$ 2$ and 4 with counterexamples. Paul Cohen [1] has constructed equations for which solutions to the Cauchy problem are not unique. First, he has constructed an equation of order $m$, with constant leading coefficients, with a real characteristic of multiplicity $s$, and with a lower order term of order $m-s+1$ with $C^{\infty}$ coefficients for which uniqueness fails. Second, he has constructed an equation of order $m$, with constant leading coefficients, with a complex characteristic of multiplicity $r$, and with a lower order term of order $m-1$ with $C^{k}$ coefficients, $k<r-2$ for which uniqueness fails.

In this second case, he claims that the lower order term can be chosen to be of order $m-b$ with $C^{k}$ coefficients, $k<r-b-1$. Although this generalization is not proven, it is stated that the proof proceeds in the same manner as for $b=1$. Proceeding with the proof for general $b$, we find his condition for the lower order term to have $C^{k}$ coefficients becomes

$$
\lambda_{l}^{b-r}\left(\Delta t_{l}\right)^{-(r+k)} \rightarrow 0 \text { as } l \rightarrow \infty .
$$

We choose, as he does, $\lambda_{l}=l^{2}, t_{l}=c l^{-\delta}, \delta>0$ and $c>0$; note $\Delta t_{l}=t_{l}-t_{l+1}$. Since $\delta$ can be made small, the condition on $k$ becomes $k<r-2 b$. For $k=0, b<r / 2$. Hence for complex characteristics, Cohen has constructed a counterexample with a lower order term of order $m-b=m-[(r+1) / 2]+1$ with $C^{0}$ coefficients.

In comparing these counterexamples with the results of $\$ \$ 2$ and 4 , we see that if a lower order derivative of order higher than we allowed in the right side of the differential inequalities (2.1) or (4.1) should occur, then uniqueness fails.

6. Uniqueness results for equations of a special form. In $\$ 2$ we have proven uniqueness of the Cauchy problem for

$$
P(D) u=\sum_{|\alpha| \leqq m-q} a_{\alpha}(x) D_{\alpha} u, \quad q=\max (s,[(r+1) / 2]) .
$$

The coefficients $a_{\alpha}(x)$ are merely required to be locally bounded and measurable. If we allow a term of order greater than $m-q$ on the right side in (6.1) then uniqueness fails as was seen in $§ 5$. 
However one may ask if uniqueness would hold when terms of order greater than $m-q$ are allowed on the right side in (6.1) provided their coefficients $a_{\alpha}(x)$ are sufficiently smooth. Plis [6] has constructed the following elliptic equation for which uniqueness fails:

$$
\left(\frac{\partial u}{\partial t}-i \frac{\partial u}{\partial x}\right)^{6}+i t^{6} \frac{\partial^{5} u}{\partial x^{5}}-\frac{\partial^{4} u}{\partial x^{4}}=f u, \quad f \in C^{\infty} .
$$

In this section we shall prove uniqueness for certain equations of the type (6.1), which include terms on the right side of order greater than $m-q$ but with constant coefficients. We shall study solutions of the differential equation

$$
Q(D) u \equiv P(D) u-\sum_{m-s \leqq|\alpha| \leqq s} c_{\alpha} D_{\alpha} u=\sum_{|\alpha|<m-s} a_{\alpha}(x) D_{\alpha} u,
$$

where $P(D)$ is a homogeneous differential operator of order $m$ with constant coefficients, with complex characteristics of multiplicity at most $r$ and real characteristics of multiplicity at most $s,[(r+1) / 2] \leqq s<m . c_{\alpha}$ are constants and $a_{\alpha}(x)$ are locally bounded measurable functions.

Now $Q^{(\alpha)}(\zeta)=P^{(\alpha)}(\zeta)$ for $|\alpha| \geqq s+1$, and hence an inequality analogous to (2.2) can be proven with $Q$ and $s+1$ replacing $P$ and $s$ respectively in (2.2). Using that inequality a Carleman type estimate analogous to Theorem 1 can be proven with $Q$ and $s+1$ replacing $P$ and $s$ respectively. Therefore let $u \in C^{m}$ be a solution of (6.3) which vanishes for $x_{1} \leqq \varepsilon\left(x_{2}^{2}+\cdots+x_{n}^{2}\right)$, for some $\varepsilon>0$, when $x$ is in a neighborhood of the origin. By using the Carleman type estimate for $Q$ and following the proof of Theorem 2, it follows that $u$ vanishes in a neighborhood of the origin.

Some examples of equations of type (6.3) are:

(1) Equation (6.2) with the coefficient of $\partial^{5} u / \partial x^{5}$ replaced by a constant.

$$
\begin{gathered}
P(D) u=\sum_{0<|\alpha|<m} c_{\alpha} D_{\alpha} u+a(x) u . \\
P(D) u=\sum_{d-1 \leq|\alpha| \leqq m-d+1} c_{\alpha} D_{\alpha} u+\sum_{|\alpha|<d-1} a_{\alpha}(x) D_{\alpha} u, \quad d=[(r+1) / 2] .
\end{gathered}
$$

\section{BIBLIOGRAPHY}

1. Paul J. Cohen, The nonuniqueness of the Cauchy problem, Office of Naval Research Technical Report No. 93, Applied Math. and Stat. Lab., Stanford Univ., 1960.

2. Peter M. Goorjian, The uniqueness of the Cauchy problem for partial differential equations which may have multiple characteristics, Dissertation, Univ. of California, Berkeley, Calif., 1969.

3. Lars V. Hörmander, (a) On the uniqueness of the Cauchy problem, Math. Scand. 6 (1958), 213-225; (b) On the uniqueness of the Cauchy problem. II, Math. Scand. 7 (1959), 177-190.

4. B. Malgrange, Sur l'unicité du problème de Cauchy, d'après A. P. Calderón, Séminaire Bourbaki, 1959, p. 178.

5. Louis Nirenberg, Uniqueness in Cauchy problems for differential equations with constant leading coefficients, Comm. Pure Appl. Math. 10 (1957), 89-105. 
6. Andrew Plis, A smooth linear elliptic differential equation without any solution in a sphere, Comm. Pure Appl. Math. 14 (1961), 599-617.

7. M. H. Protter, Unique continuation for elliptic equations, Trans. Amer. Math. Soc. 95 (1960), 81-91.

8. J. F. Trèves, Relations de domination etre opérateurs différentials, Thèse, Acta Math. 101 (1959), 1-139.

University of California,

Berkeley, California 